\title{
MARKOV-LIKE SET-VALUED FUNCTIONS ON INTERVALS AND THEIR INVERSE LIMITS
}

\author{
HAYATO IMAMURA
}

Waseda University, Japan

\begin{abstract}
We introduce Markov-like functions on intervals as a generalization of generalized Markov interval functions and define the notation of the same pattern between Markov-like functions. Then we show that two generalized inverse limits with Markov-like bonding functions having the same pattern are homeomorphic. This result gives a generalization of the results of S. Holte ([9]) and I. Banič and T. Lunder ([5]).
\end{abstract}

\section{INTRODUCTION}

Inverse sequences of continuous functions on compacta (= compact metric spaces) and inverse limits are fundamental tools of describing complicated continua and investigating dynamical systems of continuous functions. In order to study continua in 2004, Mahavier ([16]) introduced generalized inverse limits with set-valued functions on intervals. Later Ingram and Mahavier ([11]) generalized the notation to set-valued functions on compact metric spaces as follows.

Definition 1.1. For any $n \in \mathbb{N}$, let $X_{n}$ be a compact metric space and let $2^{X_{n}}$ be the collection of all nonempty closed sets of $X_{n}$. Let $f_{n}: X_{n+1} \rightarrow 2^{X_{n}}$. An inverse sequence is defined as a sequence of pairs $X_{n}$ and $f_{n}$, which is denoted by $\left\{X_{n}, f_{n}\right\}_{n \in \mathbb{N}^{*}}$. The generalized inverse limit $\lim _{\longleftarrow}\left\{X_{n}, f_{n}\right\}$ of the inverse sequence $\left\{X_{n}, f_{n}\right\}_{n \in \mathbb{N}}$ is defined by

$$
\lim _{\longleftarrow}\left\{X_{n}, f_{n}\right\}:=\left\{\left(x_{1}, x_{2}, \ldots\right) \in \prod_{n=1}^{\infty} X_{n} \mid x_{n} \in f_{n}\left(x_{n+1}\right) \text { for any } n \in \mathbb{N}\right\} .
$$

2010 Mathematics Subject Classification. 54F15, 54C60.

Key words and phrases. Inverse limits, upper semi-continuous functions, Markov maps, Markov-like functions. 
$\lim \left\{X_{n}, f_{n}\right\}$ is a subspace of $\prod_{n=1}^{\infty} X_{n}$ endowed with the product topology. In $\overleftarrow{\text { the }}$ case where $X_{n}=X$ and $f_{n}=f$ for all $n \in \mathbb{N}$, we write the inverse limit by $\lim _{\longleftarrow}\{X, f\}$.

It is known that $\lim \left\{X_{n}, f_{n}\right\}$ is compact if each $f_{n}$ is upper-semi continuous.

Markov maps on intervals are introduced as a special case of piecewise monotonic maps with the set of their critical points is a invariant set, e.g. tent maps and quadratic maps. In 2002 S. E. Holte ([9]) introduced the notation of the same pattern between Markov interval maps, and showed that any two inverse limits of inverse sequences whose bonding maps are Markov interval maps with the same pattern are homeomorphic. There are many generalizations of Markov interval maps ([1,2,5,7,17]). In 2013 I. Banič and T. Lunder ([5]) extended the notation of Markov interval maps to upper-semi continuous functions having interval-valued images on finitely many points of their domain, named generalized Markov interval functions. They also defined the notation of the same pattern between generalized Markov interval functions and showed the corresponding theorem for generalized inverse limits to Holte's theorem.

In this paper we will introduce Markov-like functions as a generalization of generalized Markov interval functions and define the notation of the same pattern between Markov-like functions. A Markov-like function allows to have finite values on each component of the complement of finitely many points, and its graph may be disconnected. Generalized inverse sequences of Markovlike functions yield interesting example. For example, the function $f$ in $[10$, Example 2.11] is a Markov-like function and the generalized inverse limit $M$ is the Hurewicz continuum. This example is studied in $[8,13,14]$. In section 3 , we will prove that any two generalized inverse limits with Markov-like bonding functions having the same pattern are homeomorphic. Consequently we can have a generalization of $[5,9]$.

Moreover, in section 4, we will show that every generalized inverse limit with Markov-like functions is one-dimensional. In section 5 we will give examples of Markov-like functions such that they do not have the same pattern but their generalized inverse limits are homeomorphic.

\section{Definition And Notation}

In this paper we may assume that all spaces are compacta and use the following notation: For any continuous map $f: X \rightarrow Y$, let $2^{f}$ : $2^{X} \rightarrow 2^{Y}$ be the induced map of $f$ given by $2^{f}(A)=f(A)$, and $G(f):=$ $\{(x, y) \in X \times Y \mid y=f(x)\}$ be the graph of $f$. Moreover, for any set-valued function $g: X \rightarrow 2^{Y}$, let $G(g):=\{(x, y) \in X \times Y \mid y \in g(x)\}$ be the graph of $g$. 
Definition 2.1. Fix $m \in \mathbb{N}_{\geq 2}$. Let $\mathbb{I}=\left[a_{1}, a_{m}\right]$ be a closed interval. Let $A: a_{1}<a_{2}<\cdots<a_{m}$ be a finite partition of $\mathbb{I}$. A set-valued function $f: \mathbb{I} \rightarrow 2^{\mathbb{I}}$ having a surjective graph is Markov-like with respect to $A$ if the following conditions are satisfied.

(1) For each $j \in\{1, \ldots, m\}$, there exist $\frac{s_{j}}{2}$ mutually disjoint closed intervals (they can be degenerate) $\left[a_{r_{1}(j)}, a_{r_{2}(j)}\right], \ldots,\left[a_{r_{s_{j}-1}(j)}, a_{r_{s_{j}}(j)}\right]$ such that

$$
\begin{gathered}
f\left(a_{j}\right)=\bigcup_{k=1}^{s_{j} / 2}\left[a_{r_{2 k-1}(j)}, a_{r_{2 k}(j)}\right], \text { and } \\
a_{r_{l}(j)} \in A \text { for each } l \in\left\{1, \ldots, s_{j}\right\} .
\end{gathered}
$$

(2) For each $j \in\{1, \ldots, m-1\}$, there exist $n_{f}(j)$ strictly monotone continuous functions $f_{j}^{1}, f_{j}^{2}, \ldots, f_{j}^{n_{f}(j)}$ defined on $\left[a_{j}, a_{j+1}\right]$ such that for each $j=1,2, \ldots, m-1$,

$$
G\left(\left.f\right|_{\operatorname{Int}\left(\left[a_{j}, a_{j+1}\right]\right)}\right)=\bigcup_{k=1}^{n_{f}(j)} G\left(\left.f_{j}^{k}\right|_{\operatorname{Int}\left(\left[a_{j}, a_{j+1}\right]\right)}\right),
$$

and for each $1 \leq k \leq n_{f}(j)$,

$$
f_{j}^{k}\left(a_{j}\right) \in f\left(a_{j}\right) \cap A \text { and } f_{j}^{k}\left(a_{j+1}\right) \in f\left(a_{j+1}\right) \cap A .
$$

Moreover, if $k_{1} \neq k_{2}$ then

$$
G\left(\left.f_{j}^{k_{1}}\right|_{\operatorname{Int}\left(\left[a_{j}, a_{j+1}\right]\right)}\right) \cap G\left(\left.f_{j}^{k_{2}}\right|_{\operatorname{Int}\left(\left[a_{j}, a_{j+1}\right]\right)}\right)=\emptyset .
$$

The graph of each Markov-like function is the union of finite arcs. Hence each Markov-like function is upper semi-continuous.

Definition 2.2. Let $\mathbb{I}=\left[a_{1}, a_{m}\right]$ and $\mathbb{J}=\left[b_{1}, b_{m}\right]$ be closed intervals and $A: a_{1}<a_{2}<\cdots<a_{m}$ and $B: b_{1}<b_{2}<\cdots<b_{m}$ be partitions of $\mathbb{I}$ and $\mathbb{J}$ respectively. A Markov-like function $f: \mathbb{I} \rightarrow 2^{\mathbb{I}}$ with respect to $A$ and a Markov-like function $g: \mathbb{J} \rightarrow 2^{\mathbb{J}}$ with respect to $B$ have the same pattern if the following conditions are satisfied.

(3) For each $j \in\{1, \ldots, m\}$,

$$
f\left(a_{j}\right) \supseteq\left[a_{r_{1}(j)}, a_{r_{2}(j)}\right] \text { if and only if } g\left(b_{j}\right) \supseteq\left[b_{r_{1}(j)}, b_{r_{2}(j)}\right] .
$$

(4) For each $j \in\{1, \ldots, m-1\}, n_{f}(j)=n_{g}(j)$ and there exists a bijection $\phi_{j}:\left\{1, \ldots, n_{f}(j)\right\} \rightarrow\left\{1, \ldots, n_{g}(j)\right\}$ such that

$$
\begin{gathered}
f_{j}^{k}\left(a_{j}\right)=a_{l_{1}(j)} \quad \text { if and only if } g_{j}^{\phi_{j}(k)}\left(b_{j}\right)=b_{l_{1}(j)}, \\
f_{j}^{k}\left(a_{j+1}\right)=a_{l_{2}(j)} \quad \text { if and only if } g_{j}^{\phi_{j}(k)}\left(b_{j+1}\right)=b_{l_{2}(j)} .
\end{gathered}
$$

The following figure is an example of the graphs of Markov-like bonding functions $f_{i}$ and $g_{i}$ having the same pattern. 

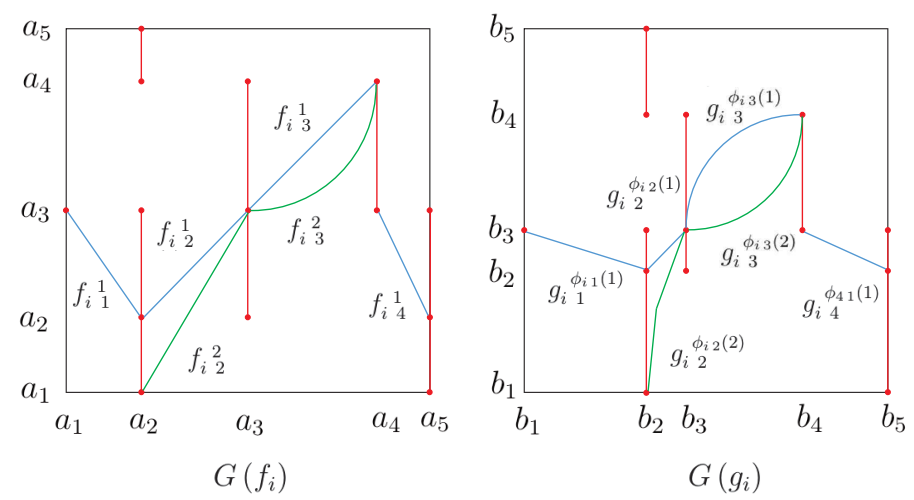

FiguRE 1. Markov-like functions having the same pattern where $m=5$

\section{MAin Theorem}

Theorem 3.4 is the main result of this paper. To prove that, we need Theorem 3.3. We can prove Theorem 3.3 with Lemma 3.1 and 3.2.

Lemma 3.1. Let $\mathbb{I}=\left[a_{1}, a_{m}\right]$ be a closed interval and $A: a_{1}<\cdots<a_{m}$ be a partition of $\mathbb{I}$, where $m \geq 2$. Let $\mathbb{J}=[0,1]$. Let $f: \mathbb{I} \rightarrow 2^{\mathbb{I}}$ be a Markov-like function with respect to $A$. Put a piecewise linear homeomorphism $h: \mathbb{I} \rightarrow \mathbb{J}$ such that $h\left(a_{1}\right)=0, h\left(a_{m}\right)=1$. Define $b_{j}=h\left(a_{j}\right)$ for each $j=1, \ldots, m$ and take a partition $B: 0=b_{1}<\cdots<b_{m}=1$ of $\mathbb{J}$. Then there is a Markov-like function $g: \mathbb{J} \rightarrow 2^{\mathbb{J}}$ with respect to $B$ such that

$$
\begin{aligned}
& \qquad 2^{h} \circ f=g \circ h \text {, and } \\
& f \text { and } g \text { have the same pattern. }
\end{aligned}
$$

Proof. Let $\alpha$ be the inverse map of $h$. Let $g: \mathbb{J} \rightarrow 2^{\mathbb{J}}$ be defined by

$$
g(x):=2^{h} \circ f \circ \alpha(x) \quad \text { for } x \in \mathbb{J} .
$$

We have

$$
g \circ h(x)=2^{h} \circ f \circ \alpha \circ h(x)=2^{h} \circ f(x) .
$$

We will show that $g$ is Markov-like with respect to $B$. Since $f$ is Markovlike with respect to $\mathrm{A}$, for each $j \in\{1, \ldots, m\}$, there exist $\frac{s_{j}}{2}$ mutually disjoint 
closed intervals $\left[a_{r_{1}(j)}, a_{r_{2}(j)}\right], \ldots,\left[a_{r_{s_{j}-1}(j)}, a_{r_{s_{j}}(j)}\right]$ such that

$$
f\left(a_{j}\right)=\bigcup_{k=1}^{\frac{s_{j}}{2}}\left[a_{r_{2 k-1}(j)}, a_{r_{2 k}(j)}\right]
$$

where $a_{r_{l}(j)} \in A$ for each $l \in\left\{1, \ldots, s_{j}\right\}$.

By definition of $h$, for each $a_{r_{1}}, a_{r_{2}} \in A$,

$$
2^{h}\left(\left[a_{r_{1}}, a_{r_{2}}\right]\right)=\left\{h(x) \mid x \in\left[a_{r_{1}}, a_{r_{2}}\right]\right\}=\left[b_{r_{1}}, b_{r_{2}}\right] .
$$

Thus,

$$
g\left(b_{j}\right)=2^{h} \circ f \circ \alpha\left(b_{j}\right)=2^{h} \circ f\left(a_{j}\right)=\bigcup_{k=1}^{s_{j} / 2}\left[b_{r_{2 k-1}(j)}, b_{r_{2 k}(j)}\right] .
$$

Fix $j \in\{1, \ldots, m-1\}$. Since $\alpha\left(\operatorname{Int}\left(\left[b_{j}, b_{j+1}\right]\right)\right)=\operatorname{Int}\left(\left[a_{j}, a_{j+1}\right]\right)$,

$$
\begin{aligned}
G\left(\left.g\right|_{\operatorname{Int}\left(\left[b_{j}, b_{j+1}\right]\right)}\right) & =\left\{(x, y) \in G\left(2^{h} \circ f \circ \alpha\right) \mid x \in \operatorname{Int}\left(\left[b_{j}, b_{j+1}\right]\right)\right\} \\
& =\bigcup_{k=1}^{n_{f}(j)}\left\{(x, y) \in G\left(h \circ f_{j}^{k} \circ \alpha\right) \mid x \in \operatorname{Int}\left(\left[b_{j}, b_{j+1}\right]\right)\right\} .
\end{aligned}
$$

Put $g_{j}^{k}:=h \circ f_{j}^{k} \circ \alpha$ for each $k \in\left\{1, \ldots, n_{f}(j)\right\}$. Then $g_{j}^{k}$ is a strictly monotone continuous function for each $k \in\left\{1, \ldots, n_{f}(j)\right\}$. Put $k_{1}, k_{2} \in$ $\left\{1, \ldots, n_{f}(j)\right\}$ with $k_{1} \neq k_{2}$. Then $G\left(\left.g_{j}^{k_{1}}\right|_{\operatorname{Int}\left(\left[b_{j}, b_{j+1}\right]\right)}\right) \cap G\left(\left.g_{j}^{k_{2}}\right|_{\operatorname{Int}\left(\left[b_{j}, b_{j+1}\right]\right)}\right)$ $=\emptyset$ since $f_{j}^{k_{1}}, f_{j}^{k_{2}}$ and $h$ are injections.

For each $k \in\left\{1, \ldots, n_{f}(j)\right\}$,

$$
\begin{aligned}
g_{j}^{k}\left(b_{j}\right) & =h \circ f_{j}^{k} \circ \alpha\left(b_{j}\right) \in 2^{h}\left(f\left(a_{j}\right) \cap A\right) \\
& =2^{h} \circ f\left(a_{j}\right) \cap 2^{h}(A)=g\left(b_{j}\right) \cap B .
\end{aligned}
$$

Similarly, $g_{j}^{k}\left(b_{j+1}\right) \in g\left(b_{j+1}\right) \cap B$. Thus $g$ is a Markov-like function with respect to $B$.

We will show that $f$ and $g$ have the same pattern. From (3.1), $f$ and $g$ satisfy the property (3) in Definition 2.2. Thus we will verify that the property (4) is satisfied. For each $j \in\{1, \ldots, m-1\}, n_{g}(j)=n_{f}(j)$ by the definition of $g$. Let us define a bijection $\phi_{j}:\left\{1, \ldots, n_{f}(j)\right\} \rightarrow\left\{1, \ldots, n_{g}(j)\right\}$ by $\phi_{j}(k)=k$. Then for each $k \in\left\{1, \ldots, n_{f}(j)\right\}$,

$$
\begin{aligned}
f_{j}^{k}\left(a_{j}\right)=a_{r_{1}(j)} & \text { if and only if } h \circ f_{j}^{k}\left(a_{j}\right)=b_{r_{1}(j)} \\
& \text { if and only if } h \circ f_{j}^{k} \circ \alpha\left(b_{j}\right)=b_{r_{1}(j)} \\
& \text { if and only if } g_{j}^{\phi_{j}(k)}\left(b_{j}\right)=b_{r_{1}(j)} .
\end{aligned}
$$

Similarly $f_{j}^{k}\left(a_{j+1}\right)=a_{r_{2}(j)}$ if and only if $g_{j}^{\phi_{j}(k)}\left(b_{j}\right)=b_{r_{2}(j)}$. Thus the property (4) is satisfied. Therefore $f$ and $g$ have the same pattern. 
Lemma 3.2. Let $\mathbb{I}$ and $\mathbb{J}$ be closed intervals. Let $\left\{f_{n}: \mathbb{I} \rightarrow 2^{\mathbb{I}}\right\}$ and $\left\{g_{n}: \mathbb{J} \rightarrow 2^{\mathbb{J}}\right\}$ be sequences of set-valued functions and let $\left\{h_{n}: \mathbb{I} \rightarrow \mathbb{J}\right\}$ be a sequence of homeomorphisms such that

$$
2^{h_{n}} \circ f_{n}=g_{n} \circ h_{n+1} \quad \text { for every } n \in \mathbb{N},
$$

where $2^{h_{n}}: 2^{\mathbb{I}} \rightarrow 2^{\mathbb{J}}$ is the induced homeomorphism by $h_{n}$. Then the generalized inverse limits $\lim _{\longleftarrow}\left\{\mathbb{I}, f_{n}\right\}$ and $\lim _{\longleftarrow}\left\{\mathbb{J}, g_{n}\right\}$ are homeomorphic.

Lemma 3.2 can be obtained in a similar way as [10, Theorem 2.9].

TheOREM 3.3. Let $\mathbb{I}=\left[a_{1}, a_{m}\right]$ be a closed interval and $A: a_{1}<\cdots<a_{m}$ be a partition of $\mathbb{I}$, where $m \geq 2$. Let $\left\{f_{n}: \mathbb{I} \rightarrow 2^{\mathbb{I}}\right\}$ be a sequence of Markovlike functions with respect to $A$. Suppose that $h: \mathbb{I} \rightarrow \mathbb{J}=[0,1]$ is a piecewise linear homeomorphism such that $h\left(a_{1}\right)=0, h\left(a_{m}\right)=1$. Define $b_{i}=h\left(a_{i}\right)$ for each $i=1, \ldots, m$ and take a partition $B: 0=b_{1}<\cdots<b_{m}=1$ of $\mathbb{J}$. Then there exists a sequence $\left\{g_{n}: \mathbb{I} \rightarrow 2^{\mathbb{J}}\right\}$ of Markov-like functions with respect to $B$ such that $f_{n}$ and $g_{n}$ have the same pattern for each $n \in \mathbb{N}$, and the generalized inverse limits $\lim \left\{\mathbb{I}, f_{n}\right\}$ and $\lim \left\{\mathbb{J}, g_{n}\right\}$ are homeomorphic.

Proof. Let $\alpha$ is the inverse mapping of $h$. For each $i \in \mathbb{N}$, let $g_{i}: \mathbb{J} \rightarrow 2^{\mathbb{J}}$ be defined by $g_{i}:=2^{h} \circ f_{i} \circ \alpha$. From Lemma 3.1, $f_{i}$ and $g_{i}$ have the same pattern. Moreover $2^{h} \circ f_{i}=g_{i} \circ h$ for each $i \in \mathbb{N}$. Therefore, by Lemma 3.2, $\lim _{\longleftarrow}\left\{\mathbb{I}, f_{n}\right\}$ and $\lim _{\longleftarrow}\left\{\mathbb{J}, g_{n}\right\}$ are homeomorphic.

Theorem 3.4. Fix $m \in \mathbb{N}_{\geq 2}$. Let $\mathbb{I}=\left[a_{1}, a_{m}\right]$ and $\mathbb{J}=\left[b_{1}, b_{m}\right]$ be closed intervals and $A: a_{1}<a_{2}<\cdots<a_{m}$ and $B: b_{1}<b_{2}<\cdots<b_{m}$ be partitions of $\mathbb{I}$ and $\mathbb{J}$, respectively. Let $\left\{f_{n}\right\}_{n \in \mathbb{N}}$ and $\left\{g_{n}\right\}_{n \in \mathbb{N}}$ be sequences of Markovlike functions with respect to $A$ and $B$ respectively. If $f_{n}$ and $g_{n}$ have the same pattern for every $n \in \mathbb{N}$, two generalized inverse limits $\lim \left\{\mathbb{I}, f_{n}\right\}$ and $\lim _{\longleftarrow}\left\{\mathbb{J}, g_{n}\right\}$ are homeomorphic.

Proof. From Theorem 3.3, we can assume both $\mathbb{I}$ and $\mathbb{J}$ are the unit interval $[0,1]$.

Let $h: \mathbb{I} \rightarrow \mathbb{J}$ be a piecewise linear homeomorphism such that $h\left(\left[a_{j}, a_{j+1}\right]\right)$ $=\left[b_{j}, b_{j+1}\right]$ for all $j=1,2, \ldots, m-1$.

STEP 1. For any point $\mathbf{x}=\left(x_{1}, x_{2}, \ldots\right) \in \lim \left\{\mathbb{I}, f_{n}\right\}$, there exists exactly one point $\mathbf{y}=\left(y_{1}, y_{2}, \ldots\right) \in \lim \left\{\mathbb{J}, g_{n}\right\}$ with $\overleftarrow{y_{1}}=h\left(x_{1}\right)$ and satisfying the following properties for each $i \overleftarrow{\in} \mathbb{N}$ :

(1)-(i) $x_{i} \in \operatorname{Int}\left(\left[a_{j}, a_{j+1}\right]\right)$ if and only if $y_{i} \in \operatorname{Int}\left(\left[b_{j}, b_{j+1}\right]\right)$,

(2)-(i) $x_{i}=a_{j}$ if and only if $y_{i}=b_{j}$,

(3)-( $i$ ) In the case of $i \geq 2, x_{i-1}=f_{i-1 j}^{k}\left(x_{i}\right)$ if and only if $y_{i-1}=g_{i-1 j}^{\phi_{i-1 j}(k)}\left(y_{i}\right)$.

To prove the existence of $\mathbf{y}$, we show that there is a decreasing sequence of closed sets $\left\{K_{n}\right\}_{n \in \mathbb{N}}$ of the Hilbert cube such that for each $n \in \mathbb{N}$, every points of $K_{n}$ satisfy the properties (1)-(i), (2)-(i), and (3)- $(i)$ for $i=1, \ldots, n$. 
Let $y_{1}=h\left(x_{1}\right)$ and $K_{1}:=\left\{h\left(x_{1}\right)\right\} \times \prod_{j=2}^{\infty} \mathbb{J}$. By the definition of $h$, the conditions (1)-(1), (2)-(1), and (3)-(1) are satisfied for each point of $K_{1}$. We note that $K_{1}$ is non-empty and closed. Suppose we defined non-empty closed sets $K_{1} \supseteq K_{2} \supseteq \cdots \supseteq K_{n}$ such that for each $i=1, \ldots, n$, every point of $K_{i}$ satisfies the properties $(1)-(j),(2)-(j)$, and $(3)-(j)$ for each $j \leq i$. We will define $K_{n+1}$. Let $\pi_{<1, n>}: \prod_{j=1}^{\infty} \mathbb{J} \rightarrow \prod_{j=1}^{n} \mathbb{J}$ be the projection mapping to the first n-coordinates. Fix $\left(y_{1}, y_{2}, \ldots, y_{n}\right) \in \pi_{<1, n>}\left(K_{n}\right)$. We define $y_{n+1}$ by the following way.

CASE 1. Suppose $x_{n+1} \in \operatorname{Int}\left(\left[a_{j}, a_{j+1}\right]\right)$ for some $j \in\{1, \ldots, m-1\}$. By the definition of $f_{n}$, there exists $k \in\left\{1,2, \ldots, n_{f}(j)\right\}$ such that

$$
x_{n}=f_{n j}^{k}\left(x_{n+1}\right) \in f_{n j}^{k}\left(\operatorname{Int}\left(\left[a_{j}, a_{j+1}\right]\right)\right) .
$$

Then we can choose $a_{l_{1}}, a_{l_{2}} \in A$ with

$$
\left(a_{l_{1}}, a_{l_{2}}\right)=f_{n j}^{k}\left(\operatorname{Int}\left(\left[a_{j}, a_{j+1}\right]\right)\right) .
$$

Hence $y_{n} \in\left(b_{l_{1}}, b_{l_{2}}\right)$ by the assumption. Since $f_{n}$ and $g_{n}$ have the same pattern,

$$
\left(b_{l_{1}}, b_{l_{2}}\right)=g_{n j}^{\phi_{n_{j}}(k)}\left(\operatorname{Int}\left(\left[b_{j}, b_{j+1}\right]\right)\right) .
$$

Therefore, there exists a $y_{n+1} \in \operatorname{Int}\left(\left[b_{j}, b_{j+1}\right]\right)$ such that

$$
y_{n}=g_{n j}^{\phi_{n j}(k)}\left(y_{n+1}\right) .
$$

CASE 2. Suppose $x_{n+1} \in A$. Choose $a_{j} \in A$ with $x_{n+1}=a_{j}$. Let $y_{n+1}=b_{j}$. By the definition of $f_{n}$, there are $a_{r_{1}(j)}, a_{r_{2}(j)} \in A$ such that

$$
x_{n} \in\left[a_{r_{1}(j)}, a_{r_{2}(j)}\right] \subseteq f_{n}\left(a_{j}\right) .
$$

By the assumption, $y_{n} \in\left[b_{r_{1}(j)}, b_{r_{2}(j)}\right]$. Since $f_{n}$ and $g_{n}$ have the same pattern,

$$
\left[b_{r_{1}(j)}, b_{r_{2}(j)}\right] \subseteq g_{n}\left(b_{j}\right)=g_{n}\left(y_{n+1}\right) .
$$

Let $K_{n+1}:=K_{n} \cap\left(\left\{\left(y_{1}, y_{2}, \ldots, y_{n+1}\right)\right\} \times \prod_{i=n+2}^{\infty} \mathbb{J}\right)$. Then $K_{n+1}$ is a non-empty closed set and each point of $K_{n+1}$ satisfies the properties (1)-(i), (2)-(i), and (3)-(i) for $i=1, \ldots, n+1$. Thus, we can have a desired decreasing sequence of closed sets $\left\{K_{n}\right\}_{n \in \mathbb{N}}$. Then there exists $\mathbf{y} \in \bigcap_{n=1}^{\infty} K_{n}$. By the construction of $\mathbf{y}, \mathbf{y} \in \lim \left\{\mathbb{J}, g_{n}\right\}$.

We will show that $\overleftarrow{\mathbf{y}}$ is uniquely determined. Suppose that for each $\mathbf{x} \in$ $\left\{\mathbb{I}, f_{n}\right\}$, there are mutually distinct points $\mathbf{y}, \mathbf{y}^{\prime} \in \lim \left\{\mathbb{J}, g_{n}\right\}$ such that

$$
y_{1}=h\left(x_{1}\right)=y_{1}^{\prime}
$$

and both points satisfy the properties (1)-(i), (2)-(i), and (3)-(i) for each $i \in \mathbb{N}$. We may assume that there exists $i_{0} \in \mathbb{N}_{\geq 2}$ such that $y_{i_{0}} \neq y_{i_{0}}^{\prime}$ and, $y_{i}=y_{i}^{\prime}$ if $i<i_{0}$. 
CASE 1. Suppose that $x_{i_{0}} \in \operatorname{Int}\left(\left[a_{j}, a_{j+1}\right]\right)$ for some $j \in\{1, \ldots, m-1\}$. Since the property $(1)-\left(i_{0}\right)$ is satisfied, $y_{i_{0}}, y_{i_{0}}^{\prime} \in \operatorname{Int}\left(\left[b_{j}, b_{j+1}\right]\right)$. By definition of $f_{i_{0}-1}$, there exists $k \in\left\{1, \ldots, n_{f_{i_{0}-1}}(j)\right\}$ such that

From the property $(3)-\left(i_{0}\right)$,

$$
x_{i_{0}-1}=f_{i_{0}-1 j}^{k}\left(x_{i_{0}}\right) .
$$

$$
\begin{aligned}
& y_{i_{0}-1}=g_{i_{0}-1 j}^{\phi_{i_{0}-1 j}(k)}\left(y_{i_{0}}\right), \\
& y_{i_{0}-1}^{\prime}=g_{i_{0}-1 j}^{\phi_{i_{0}-1 j}(k)}\left(y_{i_{0}}^{\prime}\right) .
\end{aligned}
$$

Since $g_{i_{0}-1 j}^{\phi_{i_{0}-1 j}(k)}$ is a injective map, $y_{i_{0}-1} \neq y_{i_{0}-1}^{\prime}$. It is a contradiction.

CASE 2. Suppose that $x_{i_{0}}=a_{j} \in A$ for some $j \in\{1, \ldots, m\}$. From (2)- $\left(i_{0}\right), y_{i_{0}}=b_{j}=y_{i_{0}}^{\prime}$. It is a contradiction. Thus such a case does not occur. Since we have a contradiction in each case, $\mathbf{y}$ is uniquely determined by $\mathbf{x}$.

For any $\mathbf{x} \in \lim _{\longleftarrow}\left\{\mathbb{I}, f_{n}\right\}$, choosing the point $\mathbf{y} \in \lim _{\longleftarrow}\left\{\mathbb{J}, g_{n}\right\}$ as in Step 1, we can define the function

$$
H: \lim _{\longleftarrow}\left\{\mathbb{I}, f_{n}\right\} \rightarrow \lim _{\longleftarrow}\left\{\mathbb{J}, g_{n}\right\} .
$$

STEP 2. We show that $H$ is continuous.

We provide some lemmas to show that $H$ is continuous.

Lemma 3.5. For any $\boldsymbol{x} \in \lim \left\{\mathbb{I}, f_{n}\right\}$ and $i \in \mathbb{N}$, there exists $\delta_{i}>0$ such that for each $\boldsymbol{x}^{\prime} \in \lim _{\longleftarrow}\left\{\mathbb{I}, f_{n}\right\}$ with $d\left(\boldsymbol{x}, \boldsymbol{x}^{\prime}\right)<\delta_{i}$, at least one of the following statements hold:

(1) $x_{i+1}=x_{i+1}^{\prime}, \quad x_{i+1}, x_{i+1}^{\prime} \in A$,

(2) There exist $j \in\{1, \ldots, m\}$ and $k \in\left\{1, \ldots, n_{f_{i}}(j)\right\}$ such that

$$
\left(x_{i+1}, x_{i}\right),\left(x_{i+1}^{\prime}, x_{i}^{\prime}\right) \in G\left(f_{i j}^{k}\right) .
$$

Proof. Fix $\mathbf{x} \in \lim _{\longleftarrow}\left\{\mathbb{I}, f_{n}\right\}$ and $i \in \mathbb{N}$. Let

$$
K:=\left\{\left(k^{\prime}, j^{\prime}\right) \mid\left(x_{i+1}, x_{i}\right) \in G\left(f_{i j^{\prime}}^{k^{\prime}}\right)\right\}
$$

and

$$
L:=G\left(f_{i}\right) \backslash\left(G\left(\left.f_{i}\right|_{\left\{x_{i+1}\right\}}\right) \cup \bigcup_{\left(k^{\prime}, j^{\prime}\right) \in K} G\left(f_{i j^{\prime}}^{k^{\prime}}\right)\right) .
$$

We will show that $\left(x_{i+1}, x_{i}\right) \notin \mathrm{Cl}(L)$.

Assume that $\left(x_{i+1}, x_{i}\right) \in \mathrm{Cl}(L)$. Then there is a sequence $\left\{\left(z_{i+1}^{n}, z_{i}^{n}\right)\right\}_{n \in \mathbb{N}}$ in $L$ such that

$$
\lim _{n \rightarrow \infty}\left|x_{i}-z_{i}^{n}\right|=0, \lim _{n \rightarrow \infty}\left|x_{i+1}-z_{i+1}^{n}\right|=0 .
$$


Then we can assume that $z_{i+1}^{n} \notin A$ for every $n \in \mathbb{N}$ regardless whether $x_{i+1}$ is in $A$ or not. Thus, for each $n \in \mathbb{N}$, there exist $j^{\prime} \in\{1, \ldots, m-1\}$ and $k^{\prime} \in\left\{1, \ldots, n_{f_{i}}\left(j^{\prime}\right)\right\}$ such that

$$
\left(z_{i+1}^{n}, z_{i}^{n}\right) \in G\left(f_{i j^{\prime}}^{k^{\prime}}\right)
$$

Let

$$
K_{L}:=\left\{\left(k^{\prime}, j^{\prime}\right) \mid\left(z_{i+1}^{n}, z_{i}^{n}\right) \in G\left(f_{i j^{\prime}}^{k^{\prime}}\right) \text { for some } n \in \mathbb{N}\right\} .
$$

Since $K_{L}$ is finite, there exists $(k, j) \in K_{L}$ and an infinite subset $M$ of $\mathbb{N}$ such that

$$
\left(z_{i+1}^{m}, z_{i}^{m}\right) \in G\left(f_{i j}^{k}\right) \text { for each } m \in M .
$$

Then $\left(x_{i+1}, x_{i}\right) \in G\left(f_{i j}^{k}\right)$. It is a contradiction. Thus there exists $\delta>0$ such that

$$
\left|z_{i}-x_{i}\right|^{2}+\left|z_{i+1}-x_{i+1}\right|^{2} \geq \delta^{2} \text { for any }\left(z_{i+1}, z_{i}\right) \in L \text {. }
$$

Next we define $\delta_{i}=2^{-(i+2)} \delta$ and show that $\delta_{i}$ satisfy the condition. In fact, if

$$
d\left(\mathbf{x}, \mathbf{x}^{\prime}\right)=\sum_{j=1}^{\infty} 2^{-j}\left|x_{j}-x_{j}^{\prime}\right|<\delta_{i}
$$

then

$$
2^{-i}\left|x_{i}-x_{i}^{\prime}\right|<2^{-(i+2)} \delta \text { and } 2^{-(i+1)}\left|x_{i+1}-x_{i+1}^{\prime}\right|<2^{-(i+2)} \delta .
$$

It follows that

$$
\left|x_{i}-x_{i}^{\prime}\right|^{2}+\left|x_{i+1}-x_{i+1}^{\prime}\right|^{2}<\frac{5}{16} \delta^{2} .
$$

Therefore $\left(x_{i+1}, x_{i}\right) \notin L$.

Lemma 3.6. Choose $\boldsymbol{x}, \boldsymbol{x}^{\prime} \in \lim _{\longleftarrow}\left\{\mathbb{I}, f_{n}\right\}$ and let $\boldsymbol{y}=H(\boldsymbol{x}), \boldsymbol{y}^{\prime}=H\left(\boldsymbol{x}^{\prime}\right)$. Then we have the following:

(1) If $x_{i+1}=x_{i+1}^{\prime}$ and $x_{i+1}, x_{i+1}^{\prime} \in A$, then $y_{i+1}=y_{i+1}^{\prime}$,

(2) If there exist $j \in\{1, \ldots, m-1\}$ and $k \in\left\{1, \ldots, n_{f_{i}}(j)\right\}$ such that

$$
\left(x_{i+1}, x_{i}\right),\left(x_{i+1}^{\prime}, x_{i}^{\prime}\right) \in G\left(f_{i j}^{k}\right),
$$

then

$$
\left(y_{i+1}, y_{i}\right),\left(y_{i+1}^{\prime}, y_{i}^{\prime}\right) \in G\left(g_{i j}^{\phi_{i j}(k)}\right) .
$$

Proof. This lemma is obtained directly from the definition of $H$.

Definition 3.7. Fix $i \in \mathbb{N}$. For any $\left(y_{i+1}, y_{i}\right) \in G\left(g_{i}\right), G_{\left(y_{i+1}, y_{i}\right)}\left(g_{i}\right)$ is the subset of $G\left(g_{i}\right)$ consisting of points $\left(y_{i+1}^{\prime}, y_{i}^{\prime}\right) \in G\left(g_{i}\right)$ which satisfy at least one of the following conditions:

1. $y_{i+1}=y_{i+1}^{\prime}$, or

2. there exist $j \in\{1, \ldots, m-1\}$ and $k \in\left\{1, \ldots, n_{g_{i}}(j)\right\}$ such that

$$
\left(y_{i+1}, y_{i}\right),\left(y_{i+1}^{\prime}, y_{i}^{\prime}\right) \in G\left(g_{i j}^{k}\right) \text {. }
$$


To show the continuity of $H$, we need Lemma 3.9. We can easily prove Lemma 3.9 with Lemma 3.8.

Lemma 3.8. Fix $i \in \mathbb{N}$. For any $\left(y_{i+1}, y_{i}\right) \in G\left(g_{i}\right)$ and $\epsilon>0$, there exists $\delta>0$ such that if

$$
\left(y_{i+1}^{\prime}, y_{i}^{\prime}\right) \in G_{\left(y_{i+1}, y_{i}\right)}\left(g_{i}\right),\left|y_{i}-y_{i}^{\prime}\right|<\delta
$$

then

$$
\left|y_{i+1}-y_{i+1}^{\prime}\right|<\epsilon \text {. }
$$

Proof. Fix $i \in \mathbb{N}$ and $\left(y_{i+1}, y_{i}\right) \in G\left(g_{i}\right)$. For each $j \in\{1, \ldots, m-1\}$ and $k \in\left\{1, \ldots, n_{g_{i}}(j)\right\}$, the inverse map of $g_{i j}^{k}$ is uniformly continuous. Thus the statement is easily seen by the definition of $G_{\left(y_{i+1}, y_{i}\right)}\left(g_{i}\right)$.

LEMma 3.9. Fix $n \in \mathbb{N}$ and $\left(y_{1}, y_{2}, \ldots\right) \in \mathbb{I}^{\mathbb{N}}$ with $\left(y_{i+1}, y_{i}\right) \in G\left(g_{i}\right)$ for $i=1, \ldots, n-1$. For any $\epsilon>0$, there exists $\delta_{n}>0$ such that for any $\left(y_{1}^{\prime}, \ldots, y_{n}^{\prime}, \ldots\right) \in \mathbb{I}^{\mathbb{N}}$ with $\left(y_{i+1}^{\prime}, y_{i}^{\prime}\right) \in G_{\left(y_{i+1}, y_{i}\right)}\left(g_{i}\right)$ for $i=1, \ldots, n-1$, if $\left|y_{1}-y_{1}^{\prime}\right|<\delta_{n}$, then

$$
\left|y_{i+1}-y_{i+1}^{\prime}\right|<\epsilon \text { for } 1 \leq i \leq n-1
$$

We return to the proof that $H$ is continuous.

Fix $\mathbf{x} \in \lim \left\{\mathbb{I}, f_{n}\right\}$ and let $\mathbf{y}=H(\mathbf{x})$. Fix any $\epsilon>0$ and choose $n_{\epsilon} \in \mathbb{N}$ with $\sum_{i=n_{\epsilon}+1}^{\infty} 2^{-i}<\frac{\epsilon}{2}$. From Lemma 3.5 and Lemma 3.6, there exists $\delta_{n_{\epsilon}}>0$ such that if $d\left(\mathbf{x}, \mathbf{x}^{\prime}\right)<\delta_{n_{\epsilon}}$ then

$$
\left(\pi_{i} \circ H\left(\mathbf{x}^{\prime}\right), \pi_{i+1} \circ H\left(\mathbf{x}^{\prime}\right)\right) \in G_{\left(y_{i+1}, y_{i}\right)}\left(g_{i}\right) \text { for } 1 \leq i \leq n_{\epsilon}-1 .
$$

Moreover, from Lemma 3.9, there exists $\eta_{n_{\epsilon}}>0$ such that for any $\left(y_{1}^{\prime}, \ldots\right.$, $\left.y_{n_{\epsilon}}^{\prime}, \ldots\right) \in \mathbb{I}^{\mathbb{N}}$ with $\left(y_{i+1}^{\prime}, y_{i}^{\prime}\right) \in G_{\left(y_{i+1}, y_{i}\right)}\left(g_{i}\right)$ for $1 \leq i \leq n_{\epsilon}-1$, if $\left|y_{1}-y_{1}^{\prime}\right|<$ $\eta_{n_{\epsilon}}$ then

$$
\left|y_{i+1}-y_{i+1}^{\prime}\right|<\frac{\epsilon}{2 n_{\epsilon}} \text { for } 1 \leq i \leq n_{\epsilon}-1
$$

Since $h: \mathbb{I} \rightarrow \mathbb{I}$ is continuous, there exists $\delta_{n_{\epsilon}}^{\prime}>0$ such that if $d\left(\mathbf{x}, \mathbf{x}^{\prime}\right)<\delta_{n_{\epsilon}}^{\prime}$ then

$$
\left|y_{1}-\pi_{1} \circ H\left(\mathbf{x}^{\prime}\right)\right|=\left|h\left(x_{1}\right)-h\left(x_{1}^{\prime}\right)\right|<\min \left\{\frac{\epsilon}{2 n_{\epsilon}}, \eta_{n_{\epsilon}}\right\} .
$$

Thus put $\delta:=\min \left\{\delta_{n_{\epsilon}}, \delta_{n_{\epsilon}}^{\prime}\right\}$. If $d\left(\mathbf{x}, \mathbf{x}^{\prime}\right)<\delta$ then

$$
\left|y_{i}-\pi_{i} \circ H\left(\mathbf{x}^{\prime}\right)\right|<\frac{\epsilon}{2 n_{\epsilon}} \text { for } 1 \leq i \leq n_{\epsilon} .
$$


Therefore

$$
\begin{aligned}
d\left(\mathbf{y}, H\left(\mathbf{x}^{\prime}\right)\right) & =\sum_{i=1}^{\infty} 2^{-i}\left|y_{i}-\pi_{i} \circ H\left(\mathbf{x}^{\prime}\right)\right| \\
& =\sum_{i=1}^{n_{\epsilon}} 2^{-i}\left|y_{i}-\pi_{i} \circ H\left(\mathbf{x}^{\prime}\right)\right|+\sum_{i=n_{\epsilon}+1}^{\infty} 2^{-i}\left|y_{i}-\pi_{i} \circ H\left(\mathbf{x}^{\prime}\right)\right| \\
& <\frac{\epsilon}{2}+\frac{\epsilon}{2}=\epsilon .
\end{aligned}
$$

Thus, $H$ is continuous. The same proof can be applied to the inverse map of $H$. Therefore we have that $H$ is a homeomorphism.

\section{Dimension of inVERSE Limits of MARKOV-LIKE FUnCtions}

Dimension of generalized inverse limits of set-valued functions even on $[0,1]$ may be grater than one (see [10, Chapter 5]). Recently H. Kato ([12]) gave an interesting criterion for evaluating dimension of some generalized inverse limits of set-valued functions. Using the criterion, we will show that generalized inverse limits on an interval $\mathbb{I}$ with Markov-like bonding functions is one-dimensional.

Definition 4.1. Let $\left\{X_{i}\right\}_{i \in \mathbb{N}}$ be a sequence of compact metric spaces. Let $f_{i}: X_{i+1} \rightarrow 2^{X_{i}}$ be an upper-semi continuous function for each $i \in \mathbb{N}$. For each $i \in \mathbb{N}$, define $D_{1}\left(f_{i}\right)$ and $D_{1}\left(f_{i}^{-1}\right)$ as

$$
\begin{gathered}
D_{1}\left(f_{i}\right):=\left\{x \in X_{i+1} \mid \operatorname{dim} f_{i}(x) \geq 1\right\}, \\
D_{1}\left(f_{i}^{-1}\right):=\left\{y \in X_{i} \mid \operatorname{dim} f_{i}^{-1}(y) \geq 1\right\} .
\end{gathered}
$$

The following theorem is a direct consequence of [12, Corollary 3.7] and its proof.

TheOrem 4.2 ([12, Corollary 3.7]). Let $\left\{X_{i}\right\}_{i \in \mathbb{N}}$ be a sequence of 1dimensional compact metric space. Let $f_{i}: X_{i+1} \rightarrow 2^{X_{i}}$ be an upper-semi continuous function for each $i \in \mathbb{N}$. Suppose $\operatorname{dim} D_{1}\left(f_{i}\right) \leq 0$ and $D_{1}\left(f_{i}^{-1}\right)=\emptyset$ for each $i \in \mathbb{N}$. Then $\operatorname{dim} \lim \left\{X_{i}, f_{i}\right\} \leq 1$.

THEOREM 4.3. Let $\mathbb{I}=\left[a_{1}, a_{m}\right]$ be a closed interval and $A: a_{1}<a_{2}<$ $\cdots<a_{m}$ be a partition of $\mathbb{I}$. Let $f_{i}: \mathbb{I} \rightarrow 2^{\mathbb{I}}$ be a Markov-like function with respect to $A$ for each $i \in \mathbb{N}$. Then $\operatorname{dim} \lim \left\{X_{i}, f_{i}\right\}=1$.

Proof. Fix $i \in \mathbb{N}$. From the definition of Markov-like functions, if $x \in$ $D_{1}\left(f_{i}\right)$, then $x \in A$. Hence $\operatorname{dim} D_{1}\left(f_{i}\right) \leq \operatorname{dim} A=0$. Moreover, since the graph of $f_{i}$ cannot contain a graph of any constant function, $D_{1}\left(f_{i}^{-1}\right)=\emptyset$. Therefore, from Theorem 4.2, $\operatorname{dim} \lim \left\{X_{i}, f_{i}\right\} \leq 1$. 
We next show that $\operatorname{dim} \lim \left\{X_{i}, f_{i}\right\} \geq 1$. Fix $i \in \mathbb{N}$ and put a nondegenerate closed interval $\mathbb{J}_{i} \overleftarrow{\subseteq} \mathbb{I}$ with $\mathbb{J}_{i} \cap A=\emptyset$. From the definition of Markov-like functions, one of the following holds.

(1) There exists non-degenerate closed interval $\mathbb{J}_{i+1} \subseteq \mathbb{I}$ such that $\mathbb{J}_{i+1} \subseteq$ $\operatorname{Int}\left(\left[a_{j}, a_{j+1}\right]\right)$ and $\mathbb{J}_{i}=f_{i j}^{k}\left(\mathbb{J}_{i+1}\right)$ for some $j \in\{1, \ldots, m-1\}$ and $k \in\left\{1, \ldots, n_{f_{i}(j)}\right\}$.

(2) There exists degenerate interval $\mathbb{J}_{i+1}=\left\{a_{j}\right\} \subseteq A$ such that $\mathbb{J}_{i} \subseteq$ $f_{i}\left(a_{j}\right)$.

Hence we can construct an inverse sequence $\left\{\mathbb{J}_{i}, g_{i}\right\}$ such that $\mathbb{J}_{1}$ is nondegenerate closed interval with $\mathbb{J}_{1} \cap A=\emptyset$ and for each $i \in \mathbb{N}$,

- if $\mathbb{J}_{i+1}$ is non-degenerate, there exist $j$ and $k$ such that

$$
\mathbb{J}_{i}=f_{i j}^{k}\left(\mathbb{J}_{i+1}\right) \text { and } G\left(g_{i}\right)=G\left(\left.f_{i j}^{k}\right|_{\mathbb{J}_{i+1}}\right),
$$

- if $\mathbb{J}_{i+1}$ is degenerate,

$$
\mathbb{J}_{i} \subseteq f_{i}\left(\mathbb{J}_{i+1}\right) \text { and } G\left(g_{i}\right)=G\left(f_{i}\right) \cap\left(\mathbb{J}_{i+1} \times \mathbb{J}_{i}\right) .
$$

Then $\lim _{\longleftarrow}\left\{\mathbb{J}_{i}, g_{i}\right\} \subset \lim _{\longleftarrow}\left\{X_{i}, f_{i}\right\}$ is an arc. Therefore $\operatorname{dim} \lim _{\longleftarrow}\left\{X_{i}, f_{i}\right\} \geq 1$.

Theorem 4.3 says that any generalized inverse limit of Markov-like functions is one-dimensional. Thereby, as any chainable continuum is planar, a reader may suppose that any generalized inverse limit of Markov-like functions is planar. However there are examples of nonplanar generalized inverse limits with Markov-like functions in [10, Examples 2.11 and 2.15]. Example 2.15 is also studied in $[3,4,6,15]$.

\section{Some Examples}

In this section we show that there are Markov-like functions which generate homeomorphic generalized inverse limits without the same pattern. To see that, we refer to Examples 5.1, 5.2. Here we suppose that $\mathbb{I}$ means the unit interval $[0,1]$.

EXAMPLE 5.1. Let $\left\{f_{i}: \mathbb{I} \rightarrow 2^{\mathbb{I}} \mid i \in \mathbb{N}\right\}$ be a sequence of upper semicontinuous functions. Suppose that there is a sequence of strictly monotone continuous functions $\left\{g_{i}: \mathbb{I} \rightarrow \mathbb{I} \mid i \in \mathbb{N}\right\}$ such that $(0,0),(1,1) \in G\left(g_{i}\right)$ and $G\left(f_{i}\right)=G\left(g_{i}\right)$ for each $i \in \mathbb{N}$. Then $\lim _{\longleftarrow}\{\mathbb{I}, f\}$ is an arc with endpoints $(0,0, \ldots)$, and $(1,1, \ldots)$.

Example 5.2. Fix $n \in \mathbb{N}_{\geq 2}$. Suppose that $f_{1}, \ldots, f_{n}: \mathbb{I} \rightarrow \mathbb{I}$ are strictly monotone continuous functions such that

$$
i \neq j \Rightarrow G\left(f_{i}\right) \cap G\left(f_{j}\right)=\{(0,0),(1,1)\} .
$$


Let $f: \mathbb{I} \rightarrow 2^{\mathbb{I}}$ be the upper semi-continuous function defined by

$$
G(f)=\bigcup_{i=1}^{n} G\left(f_{i}\right)
$$

Then $\lim \{\mathbb{I}, f\}$ is homeomorphic to the suspension of a Cantor set.

Proof. Let $C$ be a Cantor set and let $S(C)$ be the suspension of $C$. Here the suspension $S(C)$ is the quotient space obtained from the product space $\mathbb{I} \times C$ by shrinking the top set $\{1\} \times X$ and the bottom set $\{0\} \times X$ to the points $N$ and $S$, respectively. Let $p: \mathbb{I} \times C \rightarrow S(C)$ be the natural projection and for each $(t, z) \in \mathbb{I} \times C$ the equivalence class of $(t, z)$ we write $[t, z]=p(t, z)$. Let $\Lambda_{n}:=\prod_{k \in \mathbb{N}}\{1, \ldots, n\}$ be the product space of countable copies of the $n$-point space $\{1, \ldots, n\}$. Then there exists a homeomorphism $\varphi: C \rightarrow \Lambda_{n}$. We define $h: S(C) \rightarrow \lim \{\mathbb{I}, f\}$ as follows: for any $[t, z] \in S(C)$,

$$
\begin{aligned}
h([t, z]):=\left(x_{1}, x_{2} \ldots\right) & \text { with } x_{1}=t \text { and }\left(x_{k+1}, x_{k}\right) \in G\left(f_{s_{k}}\right) \text { for each } k \in \mathbb{N}, \\
\text { where } s & =\left(s_{1}, s_{2}, \ldots\right)=\varphi(z) .
\end{aligned}
$$

In the case of $t \in(0,1), \mathbf{x}=h([t, z]) \in \underset{\lim }{\longleftarrow}\{\mathbb{I}, f\}$ is uniquely determined. Moreover

$$
h([0, z])=(0,0, \ldots), \quad h([1, z])=(1,1, \ldots) \text { for any } z \in C .
$$

Thus, $h(N)=(0,0, \ldots)$ and $h(S)=(1,1, \ldots)$. Hence $h$ is well-defined. We note that $h$ is a bijection. In fact, for each $s=\left(s_{1}, s_{2}, \ldots\right) \in \Lambda_{n}$, let denote

$$
L_{s}:=\left\{\mathbf{x} \in \prod_{k=1}^{\infty} \mathbb{I} \mid\left(x_{k+1}, x_{k}\right) \in G\left(f_{s_{k}}\right) \text { for each } k \in \mathbb{N}\right\} .
$$

Then

$$
\lim _{\longleftarrow}\{\mathbb{I}, f\}=\bigcup_{s \in \Lambda_{n}} L_{s}, \text { and } L_{s} \cap L_{s^{\prime}}=\{(0,0, \ldots),(1,1, \ldots)\} \text { if } s \neq s^{\prime} .
$$

Hence we can easily see that $h$ is bijective. Therefore to prove that $h$ is a homeomorphism we will show that $h$ is continuous.

Let take a point $\left[t_{0}, z_{0}\right] \in S(C)$ and put $\mathbf{x}=\left(x_{1}, x_{2}, \ldots\right)=h\left(\left[t_{0}, z_{0}\right]\right)$. For an arbitrary $i \in \mathbb{N}$, take any open subset $U \subseteq \mathbb{I}$ with $x_{i} \in U$. We will show that there exists an open set $O_{i} \subset S(C)$ with $\left[t_{0}, z_{0}\right] \in O_{i}$ such that $\pi_{i}\left(h\left(O_{i}\right)\right) \subset U$.

In the case of $i=1$, since $x_{1}=t_{0}$,

$$
O_{1}=\{[t, c] \in S(C) \mid t \in U\}
$$

is an open subset of $S(C),\left[t_{0}, z_{0}\right] \in O_{1}$ and $\pi_{1}\left(h\left(O_{1}\right)\right) \subset U$.

In general case $i>1$, let $\tilde{\Lambda}$ be the subset of $\prod_{k=1}^{i-1}\{1, \ldots, n\}$ consisting of points $\left(u_{1}, \ldots, u_{i-1}\right)$ which satisfy the condition:

$$
t_{0}=x_{1} \in f_{u_{1}} \circ \cdots \circ f_{u_{i-1}}(U) \text {. }
$$


By the definition of $h,\left(s_{1}, \ldots, s_{i-1}\right) \in \tilde{\Lambda}$ where $\varphi\left(z_{0}\right)=\left(s_{1}, \ldots, s_{i-1}, s_{i}, \ldots\right) \in$ $\Lambda_{n}$. Hence

$$
V:=\tilde{\Lambda} \times \prod_{k=i}^{\infty}\{1, \ldots, n\}
$$

is an open subset of $\Lambda_{n}$ and $\varphi\left(z_{0}\right) \in V$. Note that, if $x_{i}=0$, for any $\left(u_{1}, \ldots, u_{i-1}\right) \in \prod_{k=1}^{i-1}\{1, \ldots, n\}, f_{u_{1}} \circ \cdots \circ f_{u_{i-1}}\left(x_{i}\right)=0=x_{1}$. Hence $\tilde{\Lambda}=\prod_{k=1}^{i-1}\{1, \ldots, n\}$ and $V=\Lambda_{n}$. If $x_{i}=1$, we similarly have that $V=\Lambda_{n}$. In any case

$$
W:=\bigcap_{s \in V} f_{s_{1}} \circ \cdots \circ f_{s_{i-1}}(U)=\bigcap_{\left(s_{1}, \ldots, s_{i-1}\right) \in \tilde{\Lambda}} f_{s_{1}} \circ \cdots \circ f_{s_{i-1}}(U)
$$

is an open set in $\mathbb{I}$ with $t_{0}=x_{1} \in W$ having the property: for any finite sequence $\left(y_{1}, \ldots, y_{i}\right) \in \mathbb{I} \times \cdots \times \mathbb{I}$ and any $\left(s_{1}, \ldots, s_{i-1}\right) \in \tilde{\Lambda}$,

$$
y_{1} \in W \text { and } y_{k}=f_{s_{k}}\left(y_{k+1}\right)(k=1, \ldots, i-1) \Longrightarrow y_{i} \in U
$$

Then

$$
O_{i}=p\left(W \times \varphi^{-1}(V)\right)=\{[t, z] \in S(C) \mid t \in W, \varphi(z) \in V\}
$$

is an open set in $S(C)$. If $t_{0} \in(0,1)$, then we may assume that $W \subset(0,1)$. Note that if $t_{0}=0$ or $1, \varphi^{-1}(V)=C$. Hence in these cases we can take $W$ a half open interval of the form $[0, r)$ or $(r, 1]$, respectively. Thus, $O_{i}$ is an open subset of $S(C)$ such that $\left[t_{0}, z_{0}\right] \in O_{i}$ and $\pi_{i}\left(h\left(O_{i}\right)\right) \subset U$. Therefore $h$ is continuous.

Example 5.2 induces an example of two Markov-like functions $f$ and $g$ such that they do not have the same pattern but their generalized inverse limits are homeomorphic.

ExAmple 5.3. Let $l, m \in \mathbb{N}_{\geq 2}$ with $l \neq m$. Suppose that $f_{1}, \ldots, f_{l}, g_{1}, \ldots$, $g_{m}: \mathbb{I} \rightarrow \mathbb{I}$ are strictly monotone continuous functions such that

$$
\begin{aligned}
i \neq j & \Rightarrow G\left(f_{i}\right) \cap G\left(f_{j}\right)=\{(0,0),(1,1)\}, \\
i^{\prime} \neq j^{\prime} & \Rightarrow G\left(g_{i^{\prime}}\right) \cap G\left(g_{j^{\prime}}\right)=\{(0,0),(1,1)\} .
\end{aligned}
$$

Define set-valued functions $f, g: \mathbb{I} \rightarrow 2^{\mathbb{I}}$ by

$$
G(f)=\bigcup_{i=1}^{l} G\left(f_{i}\right), G(g)=\bigcup_{j=1}^{m} G\left(g_{j}\right) .
$$

Then $f$ and $g$ do not have the same pattern but their generalized inverse limits $\lim \{\mathbb{I}, f\}$ and $\lim \{\mathbb{I}, g\}$ are homeomorphic.

From the set-valued functions $f$ and $g$ in Example 5.3 we will give an example of a pair $(\varphi, \psi)$ of Markov-like functions whose graphs coincide 
with $G(f) \cup\{1\} \times \mathbb{I}$ and $G(g) \cup\{1\} \times \mathbb{I}$, respectively but they induce nonhomeomorphic generalized inverse limits $\lim _{\leftarrow}\{\mathbb{I}, \varphi\}$ and $\lim _{\leftarrow}\{\mathbb{I}, \psi\}$.

EXAmple 5.4. Let $f, g: \mathbb{I} \rightarrow 2^{\mathbb{I}}$ be the set-valued functions $f, g: \mathbb{I} \rightarrow 2^{\mathbb{I}}$ defined in Example 5.3 as in the case of $l=2$ and $m=3$. Let $\varphi, \psi: \mathbb{I} \rightarrow 2^{\mathbb{I}}$ be defined by

$$
\begin{aligned}
& \varphi(x)=\left\{\begin{array}{lll}
\{f(x)\} & \text { if } x \in[0,1) \\
{[0,1]} & \text { if } x=1 .
\end{array}\right. \\
& \psi(x)=\left\{\begin{array}{lll}
\{g(x)\} & \text { if } x \in[0,1) \\
{[0,1]} & \text { if } x=1 .
\end{array}\right.
\end{aligned}
$$

Then $\lim _{\longleftarrow}\{\mathbb{I}, \varphi\}$ and $\lim \{\mathbb{I}, \psi\}$ are not homeomorphic.

Proof. Let

$$
\begin{aligned}
& A_{0}:=\left\{\mathbf{x} \in \prod_{j \in \mathbb{N}} \mathbb{I} \mid\left(x_{j+1}, x_{j}\right) \in G(f) \text { for each } j \in \mathbb{N}\right\}, \\
& A_{1}:=\left\{\mathbf{x} \in \prod_{j \in \mathbb{N}} \mathbb{I} \mid x_{2}=1, \text { and } x_{j} \in \varphi\left(x_{j+1}\right) \text { for any } j \geq 2\right\}, \\
& B_{0}:=\left\{\mathbf{x} \in \prod_{j \in \mathbb{N}} \mathbb{I} \mid\left(x_{j+1}, x_{j}\right) \in G(g) \text { for each } j \in \mathbb{N}\right\}, \\
& B_{1}:=\left\{\mathbf{x} \in \prod_{j \in \mathbb{N}} \mathbb{I} \mid x_{2}=1, \text { and } x_{j} \in \psi\left(x_{j+1}\right) \text { for any } j \geq 2\right\} .
\end{aligned}
$$

For each $i \in \mathbb{N}_{\geq 2}$, let

$$
\begin{array}{r}
A_{i}:=\left\{\mathbf{x} \in \prod_{j \in \mathbb{N}} \mathbb{I} \mid x_{i+1}=1,\left(x_{j+1}, x_{j}\right) \in G(f)(j<i),\right. \\
\left.x_{j} \in \varphi\left(x_{j+1}\right)(j \geq i+1)\right\}, \\
B_{i}:=\left\{\mathbf{x} \in \prod_{j \in \mathbb{N}} \mathbb{I} \mid x_{i+1}=1,\left(x_{j+1}, x_{j}\right) \in G(g)(j<i),\right. \\
\left.x_{j} \in \psi\left(x_{j+1}\right)(j \geq i+1)\right\} .
\end{array}
$$


Then we can easily see that

$$
\lim _{\longleftarrow}\{\mathbb{I}, \varphi\}=\bigcup_{i=0}^{\infty} A_{i}, \lim _{\longleftarrow}\{\mathbb{I}, \psi\}=\bigcup_{i=0}^{\infty} B_{i}
$$

and

$$
A_{i_{1}} \cap A_{i_{2}}=(1,1, \ldots), B_{i_{1}} \cap B_{i_{2}}=(1,1, \ldots) \quad \text { if } i_{1} \neq i_{2} .
$$

We will consider $A_{i}$ and $B_{i}$ for each $i \in \mathbb{Z}_{\geq 0}$. By Example 5.2, $A_{0}$ and $B_{0}$ are the union of uncountable arcs with endpoints $(0,0, \ldots)$ and $(1,1, \ldots)$, respectively. For each $i \geq 1$, since $\varphi^{-1}(\{1\})=\{1\}, \pi_{<1, i+1>}: A_{i} \rightarrow \pi_{<1, i+1>}\left(A_{i}\right)$ is a homeomorphism. Moreover,

$$
\pi_{<1, i+1>}(A)=\left\{\mathbf{x} \in \prod_{j=1}^{i+1} \mathbb{I} \mid x_{i+1}=1,\left(x_{j+1}, x_{j}\right) \in G(f)(j<i)\right\}
$$

is the union of $2^{i-1}$ many arcs. These arcs have same endpoints $u_{i}=$ $(0,0, \ldots, 0,1), v_{i}=(1,1, \ldots, 1)$ and they are mutually disjoint on each point without their endpoints. Thus $A_{i}$ is the union of $2^{i-1}$ many arcs with same endpoints $p_{i}, q_{i}$ such that $j$-th coordinate of $p_{i}$ and $q_{i}$ are equal to $u_{i}$ and $v_{i}$ respectively if $j \leq i+1$, and $j$-th coordinate of $p_{i}$ and $q_{i}$ are equal to 1 respectively if $j>i+1$. These arcs are pairwise disjoint on each point without their endpoints. In a similar way, it is also seen that $B_{i}$ is the union of $3^{i-1}$ many arcs such that they have same endpoints and they are mutually disjoint on each point without their endpoints.

The point $p=(0,1,1, \ldots) \in \lim _{\longleftarrow}\{\mathbb{I}, \psi\}$ is an endpoint of $B_{2}$. Then the maximum number of arcs such that they contain $p$ as common endpoint and they only intersect on $p$ is 3 . However for each point $q \in \lim \{\mathbb{I}, \varphi\}$, the maximum number of such arcs is equal to $2^{n}$ for some $n \in \mathbb{Z}_{\geq 0}$, or countably infinite. Therefore $\lim \{[0,1], \varphi\}$ and $\lim \{[0,1], \psi\}$ are not homeomorphic.

\section{REFERENCES}

[1] L. Alvin and J. P. Kelly, Markov set-valued functions and their inverse limits, Topology Appl. 241 (2018), 102-114.

[2] I. Banič and M. Črepnjak, Markov pairs, quasi Markov functions and inverse limits, Houston J. Math. 44 (2018), 695-707.

[3] I. Banič, M. Črepnjak, M. Merhar and U. Milutinovič, Path through inverse limits, Topology Appl. 158 (2011), 1099-1112.

[4] I. Banič, M. Črepnjak, M. Merhar and U. Milutinovič, Towards the complete classification of generalized tent maps inverse limits, Topology Appl. 160 (2013), 63-73.

[5] I. Banič and T. Lunder, Inverse limits with generalized Markov interval functions, Bull. Malays. Math. Sci. Soc. 39 (2016), 839-848.

[6] W. J. Charatonik and R. P. Roe, Inverse limits of continua having trivial shape, Houston J. Math. 38 (2012), 1307-1312. 
[7] M. Črepnjak and T. Lunder, Inverse limits with countably Markov interval functions, Glas. Mat. Ser. III 51 (2016), 491-501.

[8] G. Erceg and J. Kennedy, Topological entropy on closed sets in $[0,1]^{2}$, Topology Appl. 246 (2018), 106-136.

[9] S. E. Holte, Inverse limits of Markov interval maps, Topology Appl. 123 (2002), 421-427.

[10] W. T. Ingram, An Introduction to Inverse Limits with Set-valued Functions, Springer, New York, 2012.

[11] W. T. Ingram and W. S. Mahavier, Inverse limits of upper semi-continuous set valued functions, Houston J. Math. 32 (2006) 119-130.

[12] H. Kato, On dimension and shape of inverse limits with set-valued functions, Fund. Math. 236 (2017), 83-99.

[13] K. Kawamura and J. Kennedy, Shift maps and their variants on inverse limits with set-valued functions, Topology Appl. 239 (2018), 92-114.

[14] J. P. Kelly and T. Tennant, Topological entropy of set-valued functions, Houston J. Math. 43 (2017), 263-282.

[15] J. Kennedy and V. Nall, Dynamical properties of shift maps on inverse limits with a set valued function, Ergodic Theory Dynam. Systems 38 (2018), 1499-1524.

[16] W. S. Mahavier, Inverse limits with subsets of $[0,1] \times[0,1]$, Topology Appl. 141 (2004), 225-231.

[17] B. Raines, Orbits of turning points for maps of finite graphs and inverse limit spaces, in: Proceedings of the Ninth Prague Topological Symposium, 2001, 253-263.

\section{H. Imamura}

Faculty of Science and Engineering

Waseda University

3-4-1 Ohkubo, Shinkuju-ku, Tokyo, 169-8555

Japan

E-mail: hayato-imamura@asagi.waseda.jp

Received: 25.1.2018.

Revised: 20.6.2018. 